\title{
THE LIFT AND DRAG ON A FLAT PLATE AT LOW REYNOLDS NUMBER VIA VARIATIONAL METHODS*
}

\author{
BY \\ W. E. OLMSTEAD AND D. L. HECTOR \\ Northwestern University
}

The problem of determining the low Reynolds number lift and drag on a flat plate in Oseen flow is treated by the application of a variational principle due to Levine and Schwinger. This approach circumvents the difficulty which has limited previous results to drastically low values of the Reynolds number. In particular, an asymptotic form for the drag coefficient is derived, and found to be suitable for a much larger range of this parameter.

The ease with which the variational principle can be applied rests heavily on the ability to reduce the pair of coupled integral equations which govern this problem to one integral equation. This equation and its adjoint are shown to have solutions which are quite simply related, which further facilitates the treatment of the variational problem.

1. Introduction. The steady flow of an incompressible viscous fluid past a flat plate of finite length has been studied extensively within the framework of the Oseen approximation. Major contributions have been made by Piercy and Winny [10], Tomotika and Aoi [13], Hosimoto [4], Imai [5], Tamada and Miyagi [12], Miyagi [8], and Seebass, Tamada and Miyagi [11]. The objective of these investigations has been to determine the lift and drag experienced by the plate, and, toward that end, various asymptotic expressions for both small and large Reynolds number have been obtained. The shortcoming of these results is that the region of validity for the low Reynolds number expressions is quite limited, and generally falls far short of overlap with the high Reynolds number expressions.

All previous investigations in the low Reynolds number regime have produced asymptotic expressions for lift and drag which share the same misfortune, namely a multiplicative factor of the form $(C-\log R)^{-1}$ where the constant $C$ depends on the angle of inclination of the plate to the uniform stream and $R$ is the Reynolds number. This factor has its singularity in the range $9<R<25$ (depending on the value of $C$ ), and consequently the asymptotic forms are severely restricted.

The appearance of this troublesome factor can be traced in all cases to the expansion of a modified Bessel function for small values of the argument. Generally, such an expansion is required in order to obtain an approximate solution of the governing differential or integral equation. Unfortunately, the same factor persists even when more terms of the expansion are retained.

While it seems inevitable that the solution of the approximate problem for the local lift and drag along the plate will be limited by the undesirable factor, let us remember that our interest is not in these local quantities per se, but rather in the integral of these functions over the length of the plate. Moreover, our interest in these integrated quantities, which are called functionals, leads us to inquire about the application of

*Received November 2, 1966. 
variational methods. It is the variational principle which allows us to start with a rather rough approximation of the function and achieve an improved value for the functional.

In particular, we shall consider the stationary form for the evaluation of a functional which was introduced by Levine and Schwinger [7] to estimate the amplitude of a wave diffracted by an infinite screen with an aperture. Their approach offers an escape from our present predicament because the stationary form which is used involves the ratio of integrals of the approximate solution, thereby allowing the unwanted factor to cancel.

As we shall show, the governing integral equations for flow past a flat plate of finite length can be reduced to a form suitable for application of the mentioned variational principle. Furthermore, using only a one term approximation of the integral equation, an asymptotic form for the low Reynolds number drag will be derived. This expression for the drag appears to have a greatly extended range of validity.

2. Reduction of the integral equations. The physical situation is that of the steady flow of a viscous incompressible fluid past a flat plate of length $l$. The midpoint of the plate is taken as the origin in the $x, y$ plane; the plate is inclined at an angle $\alpha$ with respect to the positive $x$-axis. The flow velocity at infinity is in the positive $x$-direction and of constant magnitude $U$. The Oseen equations are taken to be appropriate for the analysis of this problem.

The differential form of the Oseen equations is well known, and the conversion to integral form for flow past obstacles may be found in several places. For the simplified two dimensional integral formulation applicable to this problem, we refer the reader to Miyagi [8] or Olmstead [9]. Imposing the no slip boundary conditions on the integral form of the Oseen equations leads to the following pair of coupled integral equations,

$$
\begin{aligned}
& \int_{-1}^{1}\left\{\left[\frac{1}{|\beta|(s-t)}-\operatorname{sgn}(s-t) e^{\beta(\theta-t) \cos \alpha} K_{1}(|\beta||s-t|)\right] \cos \alpha\right. \\
& \left.\quad-e^{\beta(s-t) \cos \alpha} K_{0}(|\beta||s-t|)\right\} \sigma_{1}(t) d t \\
& \quad+\int_{-1}^{1}\left[\frac{1}{|\beta|(s-t)}-\operatorname{sgn}(s-t) e^{\beta(s-t) \cos \alpha} K_{1}(|\beta||s-t|)\right] \sin \alpha \sigma_{2}(t) d t=1 \\
& \quad-1 \leq s \leq 1 \\
& \int_{-1}^{1}\left[\frac{1}{|\beta|(s-t)}-\operatorname{sgn}(s-t) e^{\beta(s-t) \cos \alpha} K_{1}(|\beta||s-t|)\right] \sin \alpha \sigma_{1}(t) d t \\
& \quad-\int_{-1}^{1}\left\{\left[\frac{1}{|\beta|(s-t)}-\operatorname{sgn}(s-t) e^{\beta(\theta-t) \cos \alpha} K_{1}(|\beta||s-t|)\right] \cos \alpha\right. \\
& \left.\quad+e^{\beta(s-t) \cos \alpha} K_{0}(|\beta||s-t|)\right\} \sigma_{2}(t) d t=0, \quad-1 \leq s \leq 1
\end{aligned}
$$

where $\beta=U l / 4 \nu=R / 4$ and $x=l s / 2$. The quantities $K_{0}(z)$ and $K_{1}(z)$ are modified Bessel functions and $\operatorname{sgn}(z)$ denotes the signum function. The functions $\sigma_{1}(t)$ and $\sigma_{2}(t)$ are proportional to the local drag and lift along the plate, respectively. In terms of the drag coefficient $C_{D}$ and the lift coefficient $C_{L}$ for the plate, we have

$$
C_{D}=-\frac{2 \pi}{\beta} \int_{-1}^{1} \sigma_{1}(t) d t
$$




$$
C_{L}=-\frac{2 \pi}{\beta} \int_{-1}^{1} \sigma_{2}(t) d t
$$

The equations (1), (2) and definitions (3), (4) are in a form which is essentially the same as that of Miyagi.

One special remark about the notation of the integral equations (1), (2) is due. The reader will note that the quantities $|\beta|$ and $\beta$ both appear in the kernels of the integral equations. In previous work, no distinction has been made between these quantities since only $R \geq 0$ is of physical interest. However, in the analysis to follow, we will find that this distinction is essential in relating various integral equations which arise. At this point, it is enough to recognize that for $\beta \geq 0$, the above integral equations are precisely those of physical interest.

Now, in order to uncouple the system (1), (2), we introduce two new functions through the following definitions:

$$
\begin{aligned}
& 2 \sigma_{1}(t)=(1+\cos \alpha) f(t ; \beta)-(1-\cos \alpha) g(t ; \beta), \\
& 2 \sigma_{2}(t)=[f(t ; \beta)+g(t ; \beta)] \sin \alpha .
\end{aligned}
$$

If we insert these definitions into the integral equations, then multiply Eq. (2) by $(1-\cos \alpha) / \sin \alpha$ and add to Eq. (1), the following integral equation for $f(t ; \beta)$ results:

$$
\int_{-1}^{1} \mathcal{K}(s-t ; \beta) f(t ; \beta) d t=1, \quad-1 \leq s \leq 1
$$

where

$$
\begin{aligned}
\Re(s-t ; \beta)=1 /|\beta|(s-t)-\operatorname{sgn}(s-t) & \exp (\beta(s-t) \cos \alpha) K_{1}(|\beta||s-t|) \\
& -\exp (\beta(s-t) \cos \alpha) K_{0}(|\beta||s-t|) .
\end{aligned}
$$

Similarly, if instead we multiply Eq. (2) by $-(1+\cos \alpha) / \sin \alpha$ and add to Eq. (1), the following integral equation for $g(t ; \beta)$ results:

$$
\int_{-1}^{1} \mathcal{H}(s-t ; \beta) g(t ; \beta) d t=1, \quad-1 \leq s \leq 1
$$

where

$$
\begin{aligned}
\mathfrak{C}(s-t ; \beta)=1 /|\beta|(s-t)-\operatorname{sgn}(s-t) & \exp (\beta(s-t) \cos \alpha) K_{1}(|\beta||s-t|) \\
& +\exp (\beta(s-t) \cos \alpha) K_{0}(|\beta||s-t|) .
\end{aligned}
$$

Thus we have reduced the coupled pair of integral equations (1), (2) to an uncoupled pair of integral equations (7), (9). Moreover, a glance at the kernels (8), (10) indicates a great similarity, and suggests the possibility of relating $f$ and $g$.

To relate the functions $f$ and $g$, consider the following equivalent form of equation (9),

$$
\int_{-1}^{1} \mathfrak{F}(-s+t ;-\beta) g(-t ;-\beta) d t=1, \quad-1 \leq s \leq 1
$$

where we have merely renamed the variables and parameter by replacing them with their negative. But now we note that

$$
\mathfrak{H C}(-s+t ;-\beta)=-\mathfrak{K}(s-t ; \beta) .
$$


Then a comparison of Eqs. (7) and (11) yields the desired relation between $f$ and $g$, namely

$$
f(t ; \beta)=-g(-t ;-\beta)
$$

This important relation allows us to express the local drag and lift, $\sigma_{1}$ and $\sigma_{2}$, in terms of one function. The definitions (5), (6) become

$$
\begin{aligned}
& \sigma_{1}(t)=\frac{1}{2}[(1+\cos \alpha) f(t ; \beta)+(1-\cos \alpha) f(-t ;-\beta)], \\
& \sigma_{2}(t)=\frac{1}{2} \sin \alpha[f(t ; \beta)-f(-t ;-\beta)] .
\end{aligned}
$$

Furthermore the relations (3), (4) for the drag and lift coefficients can be expressed rather simply as

$$
\begin{aligned}
& C_{D}=-\frac{\pi}{\beta}[(1+\cos \alpha) I(f ; \beta)+(1-\cos \alpha) I(f ;-\beta)], \\
& C_{L}=-\frac{\pi}{\beta} \sin \alpha[I(f ; \beta)-I(f ;-\beta)]
\end{aligned}
$$

where the functional $I(f ; \beta)$ is defined as

$$
I(f ; \beta)=\int_{-1}^{1} f(t ; \beta) d t .
$$

To summarize the results so far, we have succeeded in reducing the coupled integral equations (1), (2) to the one integral equation $(7)$ for $f(t ; \beta)$ [or alternately Eq. (9) for $g(t ; \beta)$ ]. Also, we emphasize that we must consider the solution of $(7)$ for positive and negative values of $\beta$ so that the quantities of physical interest may be determined.

Let us recall that our primary interest is in the total drag and lift, or $C_{D}$ and $C_{L}$. Consequently, we shall devote our efforts to obtaining a low Reynolds number approximation of the functional $I(f ; \beta)$. Toward this end, we now proceed to the application of variational methods.

3. Application of variational methods. Before we can realize a stationary form for the functional $I(f ; \beta)$, we must first notice that our integral equation of interest

$$
\int_{-1}^{1} \mathcal{K}(s-t ; \beta) f(t ; \beta) d t=1, \quad-1 \leq s \leq 1
$$

is not self-adjoint, that is $\mathscr{K}(s-t ; \beta) \neq \nVdash(t-s ; \beta)$. Thus we introduce the adjoint problem,

$$
\int_{-1}^{1} \Re(t-s ; \beta) h(t ; \beta) d t=1, \quad-1 \leq s \leq 1 .
$$

The stationary form, which we shall introduce presently, will involve both $f$ and $h$; therefore, we will find it expedient to show that these two functions can be simply related. Consider the following equivalent form of the adjoint problem

$$
\int_{-1}^{1} \Re(-t+s ; \beta) h(-t ; \beta) d t=1, \quad-1 \leq s \leq 1,
$$

where we have replaced the independent variables with their negatives. Comparison of Eqs. (7) and (20) yields the desired relation,

$$
f(t ; \beta)=h(-t ; \beta)
$$


Thus, even though our problem is not self-adjoint, we need only concern ourselves with the integral equation (7).

From the above relations, one can easily see that

$$
I(f ; \beta)=\int_{-1}^{1} f(t ; \beta) d t=\int_{-1}^{1} h(t ; \beta) d t=\int_{-1}^{1} \int_{-1}^{1} \mathcal{K}(s-t ; \beta) f(t ; \beta) h(s ; \beta) d t d s
$$

from whence follows the stationary form

$$
I(f ; \beta)=\frac{\int_{-1}^{1} f(t ; \beta) d t \int_{-1}^{1} h(t ; \beta) d t}{\int_{-1}^{1} \int_{-1}^{1} \mathscr{K}(s-t ; \beta) f(t ; \beta) h(s ; \beta) d t d s} .
$$

The reader can easily verify that, for independent small variations of $f$ and $h$ about their exact values, the expression (23) is stationary about the exact value for $I$. A form of this type was first used with great success by Levine and Schwinger [7] for the diffracted amplitude for an aperture in an infinite screen.

For a simpler form of (23) involving only the function $f$, we have

$$
I(f ; \beta)=\frac{\left[\int_{-1}^{1} f(t ; \beta) d t\right]^{2}}{\int_{-1}^{1} \int_{-1}^{1} \mathcal{K}(s-t ; \beta) f(t ; \beta) f(-s ; \beta) d t d s} .
$$

To see the utility of this expression, we need only recall the difficulty mentioned earlier, namely that previous analytical results have been severely limited by a troublesome multiplicative factor. Here we see that, even though an approximation of $f$ might contain the unwanted factor, it will not necessarily appear in the determination of $I$.

Equipped with the stationary form (24), our task remains to choose an approximation to $f$ for low Reynolds number, and carry out the evaluation of $I$.

4. Low Reynolds number results for the drag. In order to apply the stationary form (24), we first must select an approximation of $f$. For simplicity of presentation, we shall consider only a one term approximation; but even this will be seen to provide a significant improvement over previous results for the drag. A similar improvement for the lift does not follow, since the one term approximation is less adequate in representing the form of the local lift.

In the traditional way, we expand the kernel $\Re(s-t ; \beta)$ for small $\beta$ to obtain an integral equation for the approximate solution of $f$, which we call $f_{0}$. One finds

$$
\begin{array}{rr}
\int_{-1}^{1}\left[\log |s-t|+\log \frac{|\beta|}{2}+\gamma-\frac{|\beta|}{\beta} \cos \alpha\right] f_{0}(t ; \beta) d t=1, & -1 \leq s \leq 1 \\
\gamma=0.577 \ldots
\end{array}
$$

which is a well-known type of integral equation solved by Carleman [2]. The solution of $(25)$ is

$$
f_{0}(t ; \beta)=a_{0}(\beta) /\left(1-t^{2}\right)^{1 / 2}
$$

We have not bothered to specify explicitly the constant $a_{0}(\beta)$, but it does include the undesirable factor $(C-\log |\beta|)^{-1}$. 
Inserting this one term approximation of $f$ into the stationary form (24) yields

$$
I\left(f_{0} ; \beta\right)=\frac{\pi^{2}}{\int_{-1}^{1} \int_{-1}^{1} \Re(s-t ; \beta)\left(1-t^{2}\right)^{-1 / 2}\left(1-s^{2}\right)^{-1 / 2} d t d s} .
$$

Now if one is too hasty and tries to evaluate (27) approximately by replacing $\mathcal{K}(s-t ; \beta)$ with a few terms of its expansion, the resulting expression would contain the factor we have tried so hard to avoid. In fact this procedure leads to a reproduction of the results of Imai or Miyagi for small Reynolds number. Thus, to avoid difficulty, we must strive for a more suitable representation of the double integral in Eq. (27).

For convenience we define

$$
\chi(R, \alpha)=-\frac{2^{1 / 2}|R|}{4 \pi^{2}} \int_{-1}^{1} \int_{-1}^{1} \mathfrak{K}\left(s-t ; \frac{1}{4} R\right)\left(1-t^{2}\right)^{-1 / 2}\left(1-s^{2}\right)^{-1 / 2} d t d s .
$$

To evaluate $\chi(R, \alpha)$, we first note that one term in the kernel is completely eliminated through the integral identity

$$
\int_{-1}^{1} \frac{d t}{(s-t)\left(1-t^{2}\right)^{1 / 2}} d t=0, \quad-1 \leq s \leq 1
$$

We are then able to express $\chi(R, \alpha)$ as

$$
\chi(R, \alpha)=-\frac{2^{1 / 2}}{\pi^{2}} \int_{-1}^{1} F(s)\left(1-s^{2}\right)^{-1 / 2} d s
$$

where

$$
F(s)=\left[\frac{d}{d s}-\frac{1}{4}(|R|+R \cos \alpha)\right] \int_{-1}^{1} \frac{e^{1 / 4 R(s-t) \cos \alpha} K_{0}\left(\frac{1}{4}|R||s-t|\right)}{\left(1-t^{2}\right)^{1 / 2}} d t .
$$

To evaluate $F(s)$, we consider its Fourier transform

$$
\hat{F}(\xi)=\int_{-\infty}^{\infty} \exp (i \xi s) F(s) d s=\frac{-i \pi^{2}\left[\xi-\frac{1}{4} i(|R|+R \cos \alpha)\right] J_{0}(\xi)}{\left[\left(\xi-\frac{1}{4} i R \cos \alpha\right)^{2}+\frac{1}{16} R^{2}\right]^{1 / 2}}
$$

which is easily computed from application of the convolution theorem and standard forms. $J_{0}(\xi)$ denotes the ordinary Bessel function.

Thus we have

$$
\chi(R, \alpha)=-\frac{2^{1 / 2}}{2 \pi^{3}} \int_{-1}^{1} \int_{-\infty}^{\infty} e^{-i \xi s} \hat{F}(\xi)\left(1-s^{2}\right)^{-1 / 2} d \xi d s
$$

But if we carry out the $s$-integration first, we find

$$
\chi(R, \alpha)=-\frac{1}{2^{1 / 2} \pi^{2}} \int_{-\infty}^{\infty} \hat{F}(\xi) J_{0}(\xi) d \xi
$$

If the integrand of this expression is separated into its real and imaginary parts, one finds that the imaginary terms are odd functions of $\xi$ and hence these integrals vanish. The real terms are even functions of $\xi$ and the integral reduces to

$$
\begin{aligned}
\chi(R, \alpha)=\int_{0}^{\infty} \frac{J_{n}^{2}(\xi)}{r} & {\left[\frac{1}{4}(|R|+R \cos \alpha)\left(r+\xi^{2}+\frac{1}{16} R^{2} \sin ^{2} \alpha\right)^{1 / 2}\right.} \\
& \left.-\xi \operatorname{sgn} R\left(r-\xi^{2}-\frac{1}{16} R^{2} \sin ^{2} \alpha\right)^{1 / 2}\right] d \xi, \quad-\pi / 2 \leq \alpha \leq \pi / 2
\end{aligned}
$$


where

$$
r=\left[\left(\xi^{2}+\frac{1}{16} R^{2} \sin ^{2} \alpha\right)^{2}+\frac{1}{4} \xi^{2} R^{2} \cos ^{2} \alpha\right]^{1 / 2} .
$$

In terms of $\chi(R, \alpha)$, our final expression for the drag coefficient becomes

$$
C_{D}(R, \alpha)=2^{1 / 2} \pi\left[\frac{1+\cos \alpha}{\chi(R, \alpha)}+\frac{1-\cos \alpha}{\chi(-R, \alpha)}\right], \quad-\frac{\pi}{2} \leq \alpha \leq \frac{\pi}{2}
$$

For the special case of $\alpha=\pi / 2$ (the plate is normal to the oncoming stream), $\chi$ takes a particularly simple form, and we have

$$
C_{D}\left(R, \frac{\pi}{2}\right)=\frac{8 \pi}{R}\left[\int_{0}^{\infty} \frac{J_{0}^{2}(\xi)}{\left(\xi^{2}+\frac{1}{16} R^{2}\right)^{1 / 2}} d \xi\right]^{-1}
$$

To obtain a series representation of the integral which appears in equation (37), we follow the approach of Bouwkamp [1] to obtain

$$
\begin{aligned}
C_{D}\left(R, \frac{\pi}{2}\right)=\frac{8 \pi^{2}}{R}[- & \left(\log \frac{R}{16}+\gamma\right) \sum_{n=0}^{\infty} \frac{\Gamma^{2}\left(n+\frac{1}{2}\right)}{(n !)^{4}}\left(\frac{R}{4}\right)^{2 n} \\
& \left.+2 \sum_{n=2}^{\infty} \sum_{m=2}^{n} \frac{(m-1) \Gamma^{2}\left(n+\frac{1}{2}\right)}{m(2 m-1)(n !)^{4}}\left(\frac{R}{4}\right)^{2 n}\right]^{-1} \quad \gamma=0.577 \ldots
\end{aligned}
$$

Thus we have achieved a low Reynolds number expression for $C_{D}$ which is not limited, as previous results have been, by an ill-behaved multiplicative factor.

Numerical values for $C_{D}$ were determined for three angles of inclination, and the results are displayed graphically in Fig. 1.

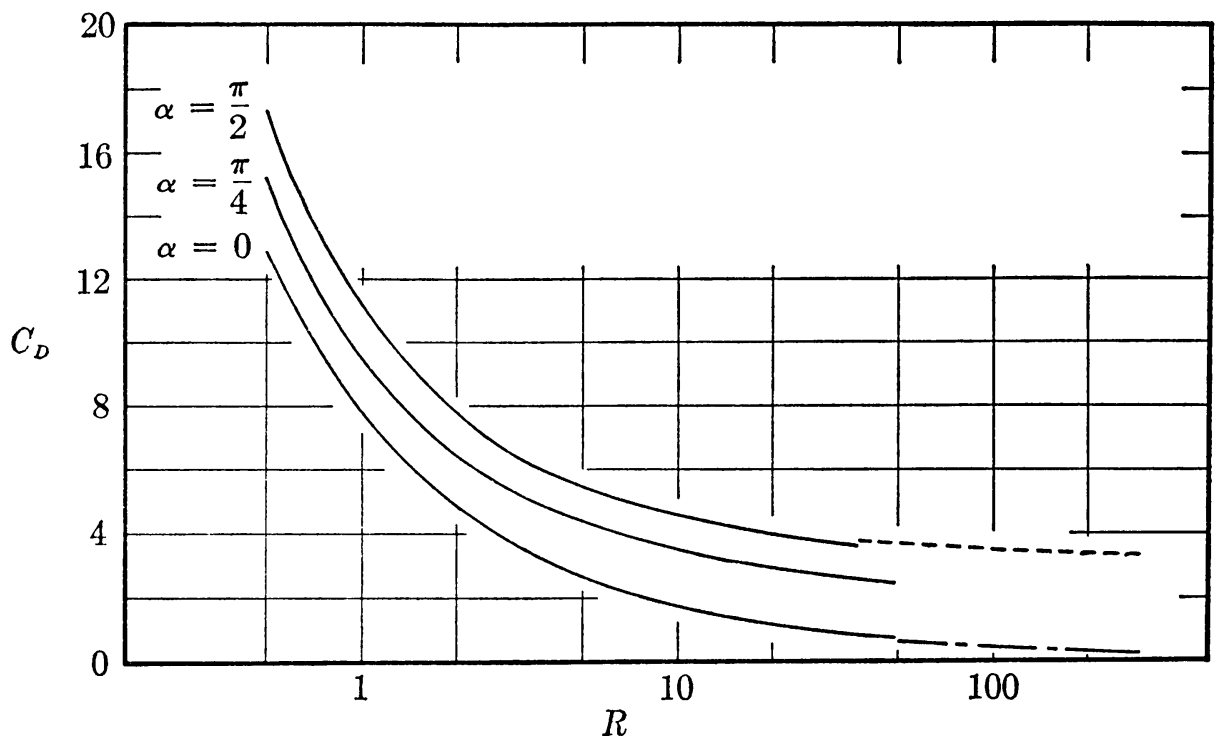

FIG. 1. Drag coefficient versus Reynolds number. Large Reynolds number results of Tamada and Miyagi (1962) _ _ _ - and Miyagi (1964)_ — - are shown. 
5. Concluding Remarks. A glance at Fig. 1 shows that our results for $C_{D}$ behave nicely, and match quite well with the large Reynolds results of others near $R=50$.

The success of our results even for relatively large values of the Reynolds number suggests that the shape (not the magnitude) of the exact solution for the local drag does not depart radically from that predicted by the approximate solution (26) in the range considered. This conjecture is borne out for the case $\alpha=0$ by the numerical results for the local drag given by Dennis and Dunwoody [3] and also the results of Seebass, Tamada and Miyagi.

While $C_{L}$ could also be calculated from the expression for $\chi(R, \alpha)$ given by (35), one finds no significant improvement over previous low Reynolds number results. The reason for this, as suggested earlier, is that the form of the local lift predicted by the one term approximation (26) is symmetrical, a clearly inadequate representation of the true situation. To achieve more adequate results for $C_{L}$ would require at least a two term approximate solution of Eq. (7).

Some insight into the limited nature of other results is provided by the expression (38) for $C_{D}(R, \pi / 2)$. Note that if the second series is neglected, we are confronted with the multiplicative factor $(\log (R / 16)+\gamma)^{-1}$.

Finally, we should point out that there are other methods, aside from variational principles, which would allow us to achieve the same final expressions for $C_{D}$. For example, if we were to calculate $a_{0}(\beta)$ in our approximate solution (26) by the Galerkin method, and then evaluate $I$ directly from its definition (18), we would obtain identical results to those presented. The equivalence of this procedure has been demonstrated by D. S. Jones [6].

The authors would like to express their gratitude to Professor I. Stakgold for his stimulating discussions on variational principles. This work was supported by the National Science Foundation, Grant GK 326.

\section{REFERENCES}

[1] C. J. Bouwkamp, Kon. Ned. Akad. Wetensch. 12, 208 (1950)

[2] E. Carleman, Math. Z. 15, 111 (1922)

[3] S. C. R. Iennis and J. Dunwoody, J. of Fluid Mech. 24, 577 (1966)

[4] H. Hosimoto, J. Phys. Soc. Japan 8, 65.3 (1953)

[5] I. Imai, Proc. Roy. Soc. A224, 141 (1954)

[6] D. S. Jones, The theory of electromagnctism, Macmillan, New York, 1964, p. 269

[7] H. Levine and J. Schwinger, Phys. Rev. 74, 958 (1948)

[8] T. Miyagi, J. Phys. Soc. Japan 19, 1063 (1964)

[9] W. E. Olmstead, J. of Math. and Phys. 44, 240 (1965)

[10] N. A. V. Piercy and H. F. Winny, Proc. Roy. Soc. A140, 543 (1933)

[11] R. Secbass, K. Tamada and T. Miyagi, Phys. of Fluids (1966)

[12] K. Tamarla and T. Miyagi, J. Phys. Soc. Japan 17, 373 (1962)

[13] S. Tomotika and T. Aoi, Quart. J. Mech. and Appl. Math. 6, 290 (1953) 